\title{
Características socioeconómicas de supervivientes de 50 o más años de edad en México de 2001 a 2012
}

\author{
Nancy Escalante Rivas, Roberto Ham Chande y \\ Carlos Echarri Cánovas
}

\author{
El Colegio de la Frontera Norte El Colegio de México
}

\section{Resumen}

El objetivo es evaluar a partir de información longitudinal y un modelo de regresión cómo antecedentes demográficos, socioeconómicos, de salud y los hábitos de toda la vida afectan la supervivencia de población de 50 años o más de edad. Hay determinantes socioeconómicos de la edad a la muerte que explican en gran medida la supervivencia de las personas en edades avanzadas, aun controlando por variables demográficas y de salud. Se esperaba que las diferencias entre los distintos niveles educativos o de ingreso se incrementaran al avanzar la edad. La posición social inicial determinada por el ingreso y la escolaridad se muestra estable y se conservan las diferencias a lo largo de las edades. No se trata de supervivencia selectiva después de los 50 años, sino que la selectividad es anterior.

Palabras clave: Envejecimiento, supervivencia, mortalidad, diferencias socioeconómicas.

\section{Abstract}

Socio-economic characteristics of population 50+ surviving from 2001 to 2012

Demographics, health condition, socio-economic status and life-styles related to life-cycle background determine mortality of the population 50 and over. A regression survival model on longitudinal variables allows estimates on the age at death. Findings show that socio-economic status largely explains survival even after controlling for demographic and health variables. Initial socio-economic status is steady and differences throughout the life-cycle are kept. The stability of the effect from income and education is not due to selective survival after age 50 but to a selection previous to that age.

Key words: Aging, survival, mortality, socio-economic status. 


\section{INTRODUCCIÓN}

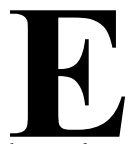

n el proceso demográfico esperado para el siglo XXI la mortalidad se desplaza hacia las edades avanzadas, lo que también podemos describir como creciente supervivencia para llegar a la vejez. De esta manera es cada vez más importante el estudio de la mortalidad en los últimos tramos de edad. En este sentido, la mortalidad debe explicarse como un efecto de todos los factores biológicos, sociales, económicos y de la salud en los ciclos de vida anteriores, esto es desde la gestación, las condiciones al nacimiento, la clase de niñez que se tuvo, cómo se pasó por la adolescencia, qué se hizo en la juventud y la vida adulta, y en qué circunstancias se llega a la vejez.

La encuesta del Estudio Nacional de Salud y Envejecimiento en México (ENASEM) permite estudiar el proceso sociodemográfico, de salud y mortalidad de los supervivientes a edades mayores aprovechando su carácter longitudinal, con rondas en 2001, 2003 y 2012. ${ }^{1}$ Este artículo se propone identificar los factores socioeconómicos que influyen, positiva o negativamente, en la supervivencia de la población de 50 años o más en el período de 2001 a 2012.

El estudio de la relación entre el nivel socioeconómico y la supervivencia en edades avanzadas se analiza bajo tres posibilidades. Una es que la desigualdad crece por acumulación de factores negativos a medida que aumenta la edad, otra es que la divergencia socioeconómica no incide debido a una selección por mortalidad, la tercera es que las diferencias relativas se mantienen constantes a lo largo de la edad. La perspectiva teórica utiliza el enfoque del curso de vida, para abordar al envejecimiento como un proceso dinámico y multidimensional, asociado a la exposición de aspectos socioeconómicos y de salud que influyen en cohortes o generaciones. El objeto principal de interés es la edad al deceso.

La hipotesis de la creciente desigualdad se propuso por Lauderdale (2001). Su análisis es por cohortes, de 1960 a 1990 a partir de los Censos de Estados Unidos, para la población blanca no institucionalizada. Sus resultados muestran que las diferencias por escolaridad también divergen entre cohortes. Asimismo, la hipótesis también se ha probado por Prus (2004) en población canadiense entre los 25 a 79 años con datos panel de

${ }^{1}$ Ya se ha levantado la ronda de 2015 cuya información todavía no estaba disponible al momento de realizar este estudio. 
"National Population Health Survey" de 1994-1995. Sus resultados encuentran relación entre el nivel socioeconómico (medido por escolaridad y el ingreso anual del hogar), y la salud (medida por índices de percepción de la salud y funcionalidad), parámetros que divergen con la edad.

Otro estudio realizado por Ross y Wu (1996) a partir de la encuesta Survey of a National Probability Simple of U.S. Households, recaba información en tres entrevistas de 1979, 1980 y 1990. Sus resultados encuentran procesos de divergencia en los grupos de escolaridad a mayor edad.

El argumento en torno a la nivelación considera al envejecimiento como un proceso que a lo largo del curso de vida funciona como un homogeneizador de la condición social, cuando los procesos biológicos terminan por dominar los determinantes sociales y finalmente la edad a la muerte es semejante independientemente del nivel socioeconómico (Liang et al., 2002).

La hipótesis sobre la redistribución hace hincapié en que la desigualdad entre las personas mayores es menos pronunciada que en grupos más jóvenes (O'Rand, 1996). Esto ha sido criticado por Mayer y Wagner (1993) quienes señalan que la nivelación es probable sí la salud, la atención a la salud y la riqueza fueran categorías relativamente independientes entre sí, lo cual no ocurre.

Esta hipótesis cuestiona las diferencias relativas causadas por factores sociales que son independientes del nivel general de la mortalidad y éstas disminuyen cuando nos acercamos a las edades "normales" de la muerte. Se añade que el estado de salud depende más de la edad que de factores sociales (Lynch, 2003). Este trabajo fue realizado para el total de la población en Estados Unidos a partir de National Health Interview Survey (NHIS) junto con National Health and Nutrition Examination Survey (NHANES) con información entre 1971 y 1992. Se encuentra convergencia después de los 30 años para la cohorte nacida en 1918 y después de los 50 años para la cohorte nacida en 1963.

Otro argumento que apoya la hipótesis sobre el desvanecimiento de las diferencias en la mortalidad entre diferentes grupos sociales, se relaciona con las condiciones de trabajo. Las personas se desenganchan de los principales sistemas de estratificación, por ejemplo, la fuerza de trabajo y por el papel tan importante que tiene el Estado de bienestar al reducir las diferencias socioeconómicas en la vejez a través de políticas redistributivas, en especial por la seguridad social (House et al., 1994). Esto significa que a lo largo del curso de vida hay una desaparición progresiva de las diferencias. En este trabajo se utiliza la encuesta longitudinal Survey En- 
titled American's Changing Lives (ACL) aunque sólo analiza 2.5 años de 1986-1989 y no incorpora población institucionalizada. Se muestra que las diferencias en las variables de salud se incrementan en las edades de 45 a 54 años y después disminuyen. Concluyen que la convergencia puede ser explicada porque los factores sociales en la vejez tienen menos impacto en relación con otros determinantes. Así se da lugar a una asociación más débil entre el nivel socioeconómico y la mortalidad en la vejez. Ross y Wu (1996) señalan que una explicación de los resultados del trabajo de House y sus colaboradores es por el mayor efecto de la educación durante la juventud, ya que es la época donde la mayor parte termina sus estudios y este efecto al avanzar la edad se difumina.

Marmot y Shipley (1996) muestran un debilitamiento del gradiente social en la mortalidad después de la jubilación para los hombres funcionarios británicos entre los 40 y 69 años, a partir del estudio "Whitehall study" con un seguimiento de 25 años para las cohortes de 1967 a 1970. Estos resultados contrastan con el caso mexicano, donde gran parte de la población no tiene acceso a los sistemas de seguridad social, lo que impide procesos de convergencia.

El estudio realizado por Liang (et al., 2002) en japoneses mayores de 60 años durante 1987 y 1999 a partir del estudio Study of Health and Wellbeing of Older Adults in Japan encuentran procesos de convergencia en el grupo de 70-79 años y concluyen que el tener una buena salud en las edades avanzadas genera un techo hasta una edad en la que quedan pocas oportunidades para mejorar la salud en promedio. Sin embargo, el contexto japonés es una sociedad muy homogénea en sus actitudes y estilos de vida mientras que la sociedad mexicana es muy heterogénea.

Una explicación teórica de la nivelación entre los distintos tipos socioeconómicos es la mortalidad selectiva. Destaca Vaupel (2001) cuando señala que todas las poblaciones son heterogéneas, algunos individuos son más frágiles que otros de forma innata o por debilidad adquirida, y por tanto sufren una mortalidad más alta en edades tempranas, lo cual deja a un subgrupo seleccionado de sobrevivientes particularmente fuertes o resistentes. Esto crea un problema fundamental para el análisis de la mortalidad en las edades avanzadas resultado del cambio en la composición de la población a lo largo del tiempo que genera una población más homogénea.

La hipótesis de las diferencias relativas constantes supone que existe una continuidad entre la condición social de las personas tanto de mediana edad como de vejez. Asimismo, tiene el apoyo empírico en los diferenciales de los salarios que se convierten a su vez en diferencias en los montos 
de las pensiones (Pampel y Hardy, 1994). Este trabajo utiliza los datos de National Longitudinal Surveys of Older Men en Estados Unidos. Comparan los resultados económicos antes y después de la edad de jubilación y no observan cambios a través de las edades. En el caso mexicano esta hipótesis sería válida por la baja cobertura de los sistemas de pensiones. Sin embargo, sería pensable en las siguientes cohortes que se jubilen con esquemas de capitalización individual porque vinculan beneficios con cotizaciones, hecho que permite una continuidad en la desigualdad a lo largo del curso de vida.

Esta hipótesis también ha sido corroborada por Hoffman (2008) a partir de la encuesta en poblacion estadounidense Health and Retirement Study (HRS) con personas mayores de 59 años analizada a lo largo de ocho años de 1992 a 2000. Se encuentran diferenciales estables entre niveles socioeconomicos en todas las edades que se explica porque los diferenciales por niveles socioeconómicos se transfieren a la salud. Finalmente, para el caso de México ninguna hipótesis se ha probado, lo que es una de las razones para plantear el presente trabajo.

\section{DATOS Y VARIABLES}

La encuesta de ENASEM se realiza en hogares donde reside al menos una persona de 50 o más años de edad. Si en un hogar residen dos o más personas en esas edades se eligen aleatoriamente una de ellas. Si la persona seleccionada está casada o unida y la pareja reside en el mismo hogar, ésta también se entrevista sin importar su edad. Se procuraron entrevistas directas y cuando esto no fue posible por razones de salud, hospitalización o ausencia temporal, se entrevistó a un informante sustituto.

En el primer panel de 2001 se entrevistaron 15186 individuos, de los cuales se tuvo noticia de 546 fallecimientos antes de la segunda ronda de entrevistas en 2003. Este segundo panel agregó 220 personas y, considerando un número menor que ya no se localizaron, se entrevistaron 14386 individuos. A partir de este panel al llegar a la tercera ronda de 2012 se notificaron 2746 fallecimientos. Sobre los decesos se hicieron entrevistas para captar las características y condiciones de los fallecidos. Este artículo es un análisis de la información de sobrevivientes y fallecidos en los periodos marcados por las tres primeras rondas de ENASEM.

Las variables utilizadas se clasifican en i) características demográficas de sexo; edad en grupos quinquenales (50-54, 55-59, 60-64, 65-69, 70$74,75-79,80-84,85$ o +); situación conyugal (nunca unido, unido, viudo y separado/divorciado) ii) hábitos de fumar y beber alcohol iii) presencia 
y número de enfermedades crónico degenerativas (diabetes, hipertensión, enfisema pulmonar, embolia cerebral, cáncer y ataque al corazón) iv) al menos una dependencia funcional (necesitar ayuda para usar el excusado, acostarse, bañarse, comer, caminar, vestirse, tomar medicamentos, manejar dinero y preparar alimentos) y v) la salud autopercibida (excelente, muy buena, buena, regular y mala).

Como estimadores del nivel socioeconómico se utilizaron el ingreso y la escolaridad. Una restricción de ENASEM es que sólo captura los ingresos del individuo y la pareja, pero no de los demás miembros del hogar. Con estos elementos la variable de ingreso se elaboró como el agregado de las percepciones personales y la media de los ingresos conjuntos con la pareja por negocios y bienes raíces. En ENASEM 2012 la media del ingreso mensual de la población mayor de 50 años masculina se estima en 3144 pesos y para las mujeres en 2086 pesos. Es importante señalar que 25 por ciento de la población mayor de 50 años declara no obtener ingresos, lo que coloca a esta población en una situación de vulnerabilidad.

Es de interés estudiar la supervivencia en función de los ingresos, ya que se ha documentado la importancia de las diferencias, incluyendo el extremo de quienes no perciben ingresos (Hoffman, 2008: 117). En este estudio se dividió la población de 50 años o más en cuartiles de ingreso. La media de ingreso del primer cuartil es de 722 pesos, la del segundo es de 1 822 pesos, en el tercero es 3726 y en el cuarto cuartil de mayor ingreso es 11 200. Los datos muestran fuertes diferencias de mortalidad entre grupos de ingreso. Es importante reconocer que el ingreso personal tiene relevancia aún en condiciones de inserción en una familia con recursos, ya que el ingreso propio otorga independencia, bienestar y autoestima a la persona envejecida. Por otra parte, el no tener ingreso individual o de pareja, provoca situaciones de desventaja familiar, social y anímica.

La variable escolaridad fue construida a partir de las características de la población mexicana, por sexo y año de nacimiento, calculada con la muestra censal de 10 por ciento en 2010. El cálculo aplica este patrón a cada individuo, "el índice puede ir desde -1 en el caso de que no tenga ningún año de escolaridad, 0 cuando tiene el número de años de instrucción promedio de la población correspondiente a su sexo y edad, a valores positivos cuando su escolaridad es superior al promedio de sus coetáneos donde el valor de 1 implica que la persona considerada estudió el doble de años que el promedio, y es 2 si estudió el triple. A partir de los valores obtenidos se calcularon los cuartiles correspondientes" (Echarri, 2008: 80). La fórmula correspondiente fue la siguiente: 


$$
\text { Escolaridad relativa }=\frac{\text { escacum }- \text { base }}{\text { base }}
$$

Siendo:

"escacum" la escolaridad acumulada.

"base" la escolaridad estándar específica para el sexo y la edad del individuo considerado.

De acuerdo con (Echarri, 2008: 80) se definió la siguiente categorización:

- Muy baja: hasta 3/4 del estándar.

- Baja: hasta 9/10 del estándar.

- Media: hasta 1.5 veces del estándar.

- Alta: más de 1.5 veces del estándar.

En este caso la escolaridad estándar es el número de años promedio de acuerdo al sexo y a la cohorte de nacimiento.

La encuesta realizada en 2001 entrevistó a 15186 personas. La encuesta de seguimiento realizada en 2003 recontactó a 89 por ciento de los individuos entrevistados en la encuesta inicial y se realizaron 546 entrevistas sobre personas fallecidas entre 2001 y 2003. Para el periodo 2003 a 2012 se partió de 14023 personas entrevistadas y se recontactó a 9 808, es decir, 89 por ciento de la muestra en 2003, se realizaron 2742 entrevistas sobre personas fallecidas entre 2003 y 2012. Los individuos que ingresan en 2012 no son sujetos de este estudio, y sólo se utiliza la línea basal 2001 y los que ingresaron en 2003.

El análisis de supervivencia tiene como unidad de análisis los años persona vividos, lo cual es la unidad de tiempo o años que aporta cada individuo de la cohorte entre las edades hasta que ocurre el evento o los años de duración del estudio. Es importante esta consideración porque la participación porcentual con respecto al número de individuos no necesariamente corresponde a la ponderación que tienen los años persona-vividos. La Tabla 1 muestra la distribución en años persona y en número de individuos que están expuestos al riesgo de muerte. En este caso es el primer levantamiento (2001-2012) y una pequeña muestra de 220 individuos que se incorporó en (2003-2012). Se consideran categorías de edad, situación conyugal, escolaridad, ingreso, salud subjetiva, dependencia funcional, número de enfermedades crónicas, hábitos de fumar y beber. 
Tabla 1: Estadísticas de la población en individuos $(\mathrm{N})$ y años persona vividos (L)

\begin{tabular}{|c|c|c|c|c|c|c|c|c|c|}
\hline \multirow[t]{2}{*}{ Variable } & \multirow[t]{2}{*}{ Categorías } & \multicolumn{4}{|c|}{ Hombres } & \multicolumn{4}{|c|}{ Mujeres } \\
\hline & & $\mathrm{N}$ & $\mathrm{L}$ & N\% & $\mathrm{L} \%$ & $\mathrm{~N}$ & $\mathrm{~L}$ & $\mathrm{~N} \%$ & $\mathrm{~L} \%$ \\
\hline \multirow{8}{*}{$\begin{array}{l}\text { Grupos } \\
\text { de edad }\end{array}$} & $50-54$ & 1494 & 76887 & 25.7 & 21.2 & 1795 & 94640 & 25.9 & 21.8 \\
\hline & $55-59$ & 1225 & 70688 & 21.0 & 19.5 & 1497 & 87282 & 21.6 & 20.1 \\
\hline & $60-64$ & 959 & 61252 & 16.5 & 16.9 & 1242 & 79437 & 17.9 & 18.3 \\
\hline & $65-69$ & 826 & 55772 & 14.2 & 15.4 & 914 & 61296 & 13.2 & 14.1 \\
\hline & $70-74$ & 592 & 42236 & 10.2 & 11.6 & 653 & 47746 & 9.4 & 11.0 \\
\hline & $75-79$ & 419 & 30679 & 7.2 & 8.5 & 447 & 33332 & 6.4 & 7.7 \\
\hline & $80-84$ & 178 & 14045 & 3.1 & 3.9 & 229 & 17498 & 3.3 & 4.0 \\
\hline & $850+$ & 131 & 11069 & 2.2 & 3.1 & 160 & 13166 & 2.3 & 3.0 \\
\hline \multirow{4}{*}{$\begin{array}{l}\text { Situación } \\
\text { conyugal }\end{array}$} & Casado & 4819 & 297664 & 82.7 & 82.1 & 3956 & 242621 & 57.0 & 55.9 \\
\hline & $\begin{array}{l}\text { Separado y } \\
\text { divorciado }\end{array}$ & 157 & 9162 & 2.7 & 2.5 & 350 & 20574 & 5.0 & 4.7 \\
\hline & Viudo & 324 & 18817 & 5.6 & 5.2 & 772 & 44963 & 11.1 & 10.4 \\
\hline & Nunca unido & 524 & 37007 & 9.0 & 10.2 & 1859 & 126239 & 26.8 & 29.1 \\
\hline \multirow[t]{4}{*}{ Escolaridad } & $\begin{array}{l}\text { Muy baja: hasta } \\
3 / 4 \text { del estándar }\end{array}$ & 3799 & 246754 & 65.2 & 68.0 & 4399 & 286808 & 63.4 & 66.0 \\
\hline & $\begin{array}{l}\text { Baja: hasta 9/10 } \\
\text { del estándar }\end{array}$ & 891 & 53040 & 15.3 & 14.6 & 1165 & 66939 & 16.8 & 15.4 \\
\hline & $\begin{array}{l}\text { Media: hasta } \\
1.5 \text { veces el } \\
\text { estándar }\end{array}$ & 466 & 28094 & 8.0 & 7.7 & 763 & 45747 & 11.0 & 10.5 \\
\hline & $\begin{array}{l}\text { Alta: más de } \\
1.5 \text { veces el } \\
\text { estándar }\end{array}$ & 668 & 34742 & 11.5 & 9.6 & 610 & 34903 & 8.8 & 8.0 \\
\hline \multirow[t]{5}{*}{ Ingreso } & $\begin{array}{l}\text { No perciben } \\
\text { ingreso }\end{array}$ & 1068 & 69462 & 18.3 & 19.2 & 2216 & 11245 & 31.9 & 33.2 \\
\hline & $1^{\circ}$ cuartil & 1231 & 84427 & 21.1 & 23.3 & 1629 & 107007 & 23.5 & 24.6 \\
\hline & $2^{\circ}$ cuartil & 927 & 59162 & 15.9 & 16.3 & 950 & 59473 & 13.7 & 13.7 \\
\hline & $3^{\circ}$ cuartil & 1287 & 77435 & 22.1 & 21.4 & 1131 & 67324 & 16.3 & 15.5 \\
\hline & $4^{\circ}$ cuartil & 1311 & 72134 & 22.5 & 19.9 & 1011 & 56348 & 14.6 & 13.0 \\
\hline \multirow[t]{3}{*}{$\begin{array}{l}\text { Salud } \\
\text { subjetiva }\end{array}$} & $\begin{array}{l}\text { Excelente, muy } \\
\text { buena, buena }\end{array}$ & 2258 & 130627 & 38.8 & 36.0 & 1942 & 115415 & 28.0 & 26.6 \\
\hline & Regular & 3392 & 221224 & 58.2 & 61.0 & 4792 & 307225 & 69.1 & 70.7 \\
\hline & Mala & 174 & 10779 & 3.0 & 3.0 & 203 & 11757 & 2.9 & 2.7 \\
\hline \multirow[t]{2}{*}{$\begin{array}{l}\text { Dependencia } \\
\text { funcional }\end{array}$} & $\begin{array}{l}\text { Sin } \\
\text { dependencia }\end{array}$ & 4887 & 297756 & 83.9 & 82.1 & 5354 & 325622 & 77.2 & 75.0 \\
\hline & $\begin{array}{l}\text { Con } \\
\text { dependencia }\end{array}$ & 937 & 64874 & 16.1 & 17.9 & 1583 & 108775 & 22.8 & 25.0 \\
\hline \multirow{5}{*}{$\begin{array}{l}\text { Número de } \\
\text { enfermedades } \\
\text { crónico- } \\
\text { degenerativas }\end{array}$} & $\begin{array}{l}\text { Ausencia de } \\
\text { enfermedad }\end{array}$ & 2844 & 174538 & 48.8 & 48.1 & 2334 & 141251 & 33.6 & 32.5 \\
\hline & 1 enfermedad & 1941 & 121818 & 33.3 & 33.6 & 2897 & 183441 & 41.8 & 42.2 \\
\hline & 2 enfermedades & 825 & 52394 & 14.2 & 14.4 & 1342 & 85239 & 19.3 & 19.6 \\
\hline & 3 enfermedades & 184 & 11859 & 3.2 & 3.3 & 318 & 21257 & 4.6 & 4.9 \\
\hline & 4 enfermedades & 30 & 2021 & 0.5 & 0.6 & 46 & 3209 & 0.7 & 0.7 \\
\hline \multirow[t]{2}{*}{ Fumar } & No fumar & 1945 & 119298 & 33.4 & 32.9 & 5171 & 327364 & 74.5 & 75.4 \\
\hline & Fumar & 3879 & 243332 & 66.6 & 67.1 & 1766 & 107033 & 25.5 & 24.6 \\
\hline \multirow[t]{3}{*}{ Beber } & Beber & 2811 & 168703 & 48.3 & 46.5 & 1185 & 70143 & 17.1 & 16.1 \\
\hline & No beber & 3013 & 193927 & 51.7 & 53.5 & 5752 & 364254 & 82.9 & 83.9 \\
\hline & Total & 5824 & 362630 & 100.0 & 100.0 & 6937 & 434397 & 100.0 & 100.0 \\
\hline
\end{tabular}

Fuente: elaboración propia a partir de ENASEM 2001 y 2003. 


\section{Metodología}

Los modelos de supervivencia responden al tema de investigación al explicar por qué ciertos sujetos tienen mayor propensión de supervivencia a lo largo del tiempo. Son modelos de regresión en los cuales el "riesgo" de supervivencia en el tiempo se estima a partir de un conjunto de variables explicativas independientes, que de modo longitudinal consideran la dinámica temporal de los eventos y los procesos que los generan. Tienen la ventaja de contrastar empíricamente aseveraciones de causalidad. Estos modelos permiten el análisis de datos incompletos, observaciones parciales, o seguimiento interrumpido. Son individuos de los que se desconoce el momento de ocurrencia del evento de interés, que en este caso es la muerte. El tiempo de seguimiento es menor al máximo posible y puede ser por dos razones principales:

1. Cuando el tiempo de seguimiento termina antes de producirse la muerte. En este caso nos referimos a los sobrevivientes del estudio para los que la duración utilizada es la edad a la última entrevista.

2. Cuando hay pérdida de seguimiento y el individuo deja de participar en el estudio que continúa. En este caso se asume que estuvieron expuestos a la supervivencia la mitad del período antes de dejar de ser observados.

El análisis de supervivencia permite trabajar con casos en los que no se ha experimentado la muerte porque de no incluirlos produciría sesgos de selección. Los casos con duraciones más cortas tenderían a prevalecer y esto subestima la edad a la muerte (Singer y Willett, 2003: 316-317).

Para planificar correctamente un estudio de supervivencia, debe definirse apropiadamente el inicio del seguimiento que en este caso es a partir de los 50 años de edad, dado que la información disponible lo permite y da cuenta del calendario a la muerte en el tránsito a las edades avanzadas. La unidad de tiempo que se utiliza son años y el evento que se analiza es la muerte. Ya que se estudia el tiempo de seguimiento, deben quedar claras las fechas de inicio del mismo y del último valor conocido para cada individuo, para así considerar el tiempo de supervivencia.

El conjunto en riesgo es el número de individuos que están expuestos a la muerte en cada edad. Se asume que el conjunto en riesgo de cada intervalo es representativo de todos los individuos que han estado en riesgo de ocurrencia (Singer y Willett, 2003: 326). 
Sea $\mathrm{T}>0$ la variable aleatoria que mide el tiempo hasta la ocurrencia de un determinado suceso. En el análisis de supervivencia hay dos distribuciones que resultan especialmente relevantes (Singer y Willett, 2003: 330).

La función de supervivencia estima la proporción acumulada de adultos mayores para los que al final de este período todavía continuaban con vida. Es la probabilidad de que un individuo sobreviva pasando un período de tiempo. La función de supervivencia tiene un valor decreciente al incrementar $t$. $\mathrm{S}(\mathrm{t})=1$ cuando $\mathrm{t}=0$ y es 0 cuando muere la última persona.

$$
\begin{aligned}
& \widehat{S}\left(t_{j}\right)=\frac{n \text { quienes no han experimentado el evento para el final del periodo }}{j} \\
& n \text { conjunto de datos } s_{j} \\
& \left(t_{i j}\right)=\operatorname{Pr}\left[T_{i}>j\right]
\end{aligned}
$$

La función de riesgo especifica la probabilidad condicional instantánea de que un individuo i experimente el evento ("muerte") en el intervalo, dado que no lo ha experimentado ("ha sobrevivido") hasta el momento t. Es decir, proporciona información de sí y cuando ocurre el evento de interés. Mientras más grande es el peligro mayor es el riesgo y cuando el peligro es bajo, menor es el riesgo. Donde no ocurren eventos, la función es constante.

$$
\begin{array}{r}
\hat{h}\left(t_{j}\right)=\frac{n \text { eventos }_{j}}{n \text { expuestos al riesgo }_{j}} \\
\left(h t_{i j}\right)=\operatorname{Pr}\left[T_{i}=j \mid T_{i} \geq j\right]
\end{array}
$$

Cuando ocurren eventos o perdida de seguimiento (censura) el cálculo de la función de riesgo se estima a partir de los que permanecen en conjunto, por lo que se requiere la función de riesgo del intervalo de todos los años previos y la función de supervivencia es el producto sucesivo del complemento de las funciones de riesgo (Singer y Willett, 336, 2003).

$$
\widehat{S}\left(t_{j}\right)=\left[1-\hat{h}\left(t_{j}\right)\right]\left[1-\hat{h}\left(t_{j-1}\right)\right]\left[1-\hat{h}\left(t_{j-2}\right)\right] \ldots\left[1-\hat{h}\left(t_{j}\right)\right]
$$

Se elige un modelo discreto ya que el tiempo en el que ocurren las muertes es discreto. Se reporta el intervalo de tiempo donde ocurrieron, provocando que en un solo intervalo, esto es en un año, existan varios empates. Las razones por las que se decide utilizar el modelo en tiempo 
discreto obedece a que es posible explorar con bastante flexibilidad las distintas formas que pueden adquirir la asociación entre el tiempo y la probabilidad de experimentar la muerte y sobre todo porque en demografía generalmente no se cuenta con datos sobre la duración exacta a la muerte, sino sobre la ocurrencia en intervalos discretos de duraciones.

Los modelos de regresión en tiempo discreto asumen que la información disponible no es sobre el tiempo exacto a la muerte, sino sobre la ocurrencia en intervalos porque el riesgo instantáneo no puede ser calculado directamente. Debe aproximarse mediante la estimación de la probabilidad de experimentar la muerte en el intervalo de tiempo en cuestión. El modelo de tiempo discreto se expresa en la siguiente ecuación de regresión logística (Singer y Willett, 2003: 371).

$$
\operatorname{logit} h\left(t_{i j}\right)=\left[\alpha_{0}+\alpha_{\text {tiempo }}+\beta_{1} X_{1 i j}+\beta_{2} X_{2 i j}+\cdots+\beta_{p} X_{p i j}\right]
$$

Donde:

$\alpha_{\text {tiempo }} \quad$ Representa cada parámetro de intercepción en el tiempo.

$\alpha_{0} \quad$ Es constante cuando todos los predictores son cero.

$\beta_{1}, \beta_{2} \ldots, \beta_{\mathrm{p}}$ Es el efecto del predictor controlado por los otros predictores.

$h\left(t_{\mathrm{ij}}\right) \quad$ La función de riesgo o probabilidad de experimentar el evento para el individuo i en el período de tiempo j. Los individuos son distinguidos entre si sobre la base de los predictores y pueden tener diferentes funciones de riesgo.

$X_{p i j} \quad$ Denota los valores del individuo $i$ por el predictor en el periodo $j$. En este caso toma el mismo valor en cada período es fija e invariante en el tiempo, reportada al inicio del estudio (Singer y Willett, 2003: 369).

Uno de los supuestos de la regresión logística en tiempo discreto es que los efectos de las variables independientes no están en función de lapsos o edades. Esto es que los momios estimados son los mismos a lo largo de todo el periodo de exposición. Este supuesto puede o no ser plausible, de modo que es importante verificar su factibilidad en cada aplicación para estimar probabilidades de muerte, comprobando que se cumple la proporcionalidad de momios en cada grupo de edades.

Comparar la razón de momios entre dos distintos grupos a las mismas edades permite mostrar que el efecto multiplicativo por variaciones del ingreso sobre el riesgo de morir es el mismo independientemente de la 
edad. Esta relación se mantiene en todos los grupos de edades y entre los distintos grupos de ingreso elegidos.

Una manera de probar este supuesto es verificar si existen o no interacciones estadísticamente significativas entre la variable de duración y la variable independiente en cuestión. Si la interacción no es estadísticamente significativa hay argumentos para sostener el supuesto de proporcionalidad. Si en cambio resulta estadísticamente significativa, entonces proporciona evidencia de que los efectos de la variable independiente dependen de la duración y por tanto no se cumple el supuesto de proporcionalidad.

Se decidió agrupar los efectos de la duración en un menor número de coeficientes, ya que resulta problemático la inclusión de una variable dicotómica para cada duración individual, o se tienen pocos casos para cada duración. Esta solución implica el supuesto de que la probabilidad de muerte entre $t$ y $t+1$ denotada como $\mathrm{q}(\mathrm{t}, \mathrm{t}+1)$ no varían al interior de cada grupo de edades/duraciones, pero sí entre grupos. Los resultados del modelo se realizaron en duraciones o edades quinquenales porque el análisis de la edad presenta un mejor ajuste y una correcta especificación reflejando adecuadamente el patrón variación por edades en los riesgos de ocurrencia a la muerte.

Se realizaron modelos binarios de regresión logística con la prueba de Wald que permite contrastar si un coeficiente B es significativamente diferente de 0 . Es decir, que tiene un aporte estadísticamente significativo a la "explicación" de la variable de respuesta. Para analizar la bondad de ajuste y la selección del mejor modelo se utilizó la prueba BIC (criterio de información bayesiano) que permite seleccionar modelos más parsimoniosos con el menor número de parámetros a partir de calcular el modelo con máximo probabilidad a posteriori y el criterio de información Akaike (AIC) que permite seleccionar un modelo tomando como base el tamaño de la muestra y el número de parámetros utilizados en los modelos. El análisis de las pruebas BIC y AIC que se desprende de la Tabla 2 muestra que la mejor bondad de ajuste la tiene el modelo 10, porque tiene los menores valores, e incluye todas las variables. Los resultados se presentan en razones de momios (denominados OR odd ratios) y se muestran dos niveles de significación estadística: p 0.05 y p 0.001 . 
Tabla 2a: Resultados modelo de regresión logística en tiempo discreto personas de 50 años o más. México 2001 y 2003-2012

\begin{tabular}{|c|c|c|c|c|c|}
\hline Variables & Modelo 1 & Modelo 2 & Modelo 3 & Modelo 4 & Modelo 5 \\
\hline & $\mathrm{RR}$ & $\mathrm{RR}$ & $\mathrm{RR}$ & $\mathrm{RR}$ & $\mathrm{RR}$ \\
\hline cons & $0.017^{b}$ & $0.019^{b}$ & $0.018^{b}$ & $0.018^{b}$ & $0.022^{b}$ \\
\hline $55-59(\operatorname{ref} 50-54)$ & 0.981 & 0.973 & 0.983 & 0.988 & 0.992 \\
\hline $60-64$ & $0.135^{b}$ & $0.137^{\mathrm{b}}$ & $0.141^{\mathrm{b}}$ & $0.142^{b}$ & $0.146^{\mathrm{b}}$ \\
\hline $65-69$ & $0.137^{\mathrm{b}}$ & $0.139^{b}$ & $0.140^{b}$ & $0.140^{\mathrm{b}}$ & $0.141^{b}$ \\
\hline $70-74$ & $0.186^{\mathrm{b}}$ & $0.188^{b}$ & $0.184^{b}$ & $0.186^{\mathrm{b}}$ & $0.181^{b}$ \\
\hline $75-79$ & $0.206^{\mathrm{b}}$ & $0.208^{b}$ & $0.196^{\mathrm{b}}$ & $0.196^{\mathrm{b}}$ & $0.188^{b}$ \\
\hline $80-84$ & $0.282^{b}$ & $0.286^{\mathrm{b}}$ & $0.259^{\mathrm{b}}$ & $0.258^{b}$ & $0.243^{b}$ \\
\hline 85 o más & $0.351^{\mathrm{b}}$ & $0.357^{\mathrm{b}}$ & $0.309^{\mathrm{b}}$ & $0.307^{\mathrm{b}}$ & 0.285 \\
\hline Sexo(ref. mujer) & & $0.811^{b}$ & $0.723^{b}$ & $0.724^{b}$ & $0.699^{\mathrm{b}}$ \\
\hline Nunca unido (ref.unidos) & & & $1.359^{\mathrm{b}}$ & $1.364^{\mathrm{b}}$ & $1.286^{\mathrm{a}}$ \\
\hline Separado, divorciado & & & $1.251^{\mathrm{a}}$ & $1.251^{\mathrm{a}}$ & $1.176^{\mathrm{a}}$ \\
\hline Viudo & & & $1.571^{b}$ & $1.570^{b}$ & $1.485^{b}$ \\
\hline Escolaridad relativa baja (ref. muy baja) & & & & $0.866^{\mathrm{a}}$ & 0.915 \\
\hline Media & & & & 0.936 & 1.018 \\
\hline Alta & & & & 1.007 & $1.198^{\mathrm{a}}$ \\
\hline 1er cuartil (ref. no percibe ingresos) & & & & & 0.956 \\
\hline 2do cuartil & & & & & 0.953 \\
\hline 3er cuartil & & & & & $0.812^{b}$ \\
\hline 4to cuartil & & & & & $0.586^{\mathrm{b}}$ \\
\hline \multicolumn{6}{|l|}{ Regular (ref. excelente, muy buena, buena) } \\
\hline \multicolumn{6}{|l|}{ Mala } \\
\hline \multicolumn{6}{|l|}{ con dependencia (ref. sin dependencia) } \\
\hline \multicolumn{6}{|l|}{$\begin{array}{l}1 \text { enfermedad (ref. ausencia de } \\
\text { enfermedad) }\end{array}$} \\
\hline \multicolumn{6}{|l|}{2 enfermedad } \\
\hline \multicolumn{6}{|l|}{3 enfermedad } \\
\hline \multicolumn{6}{|l|}{4 enfermedad } \\
\hline \multicolumn{6}{|l|}{ Beber (ref. no beber) } \\
\hline \multicolumn{6}{|l|}{ Fumar (ref. no fumar) } \\
\hline AIC & 37933.86 & 37904.15 & 37816.97 & 37815.89 & 37756.9 \\
\hline $\mathrm{BIC}$ & 37982.41 & 37958.77 & 37889.8 & 37906.93 & 37872.2 \\
\hline
\end{tabular}

$\mathrm{N}=3195 .{ }^{a} \mathrm{p} \leq 0.05 ;{ }^{b} \mathrm{p} \leq 0.001$

Fuente: elaboración propia a partir de ENASEM. 
Tabla 2b: Resultados modelo de regresión logística en tiempo discreto personas de 50 años o más. México 2001 y 2003-2012

\begin{tabular}{|c|c|c|c|c|c|}
\hline Variables & Modelo 6 & Modelo 7 & Modelo 8 & Modelo 9 & Modelo 10 \\
\hline & $\mathrm{RR}$ & RR & $\mathrm{RR}$ & $\mathrm{RR}$ & $\mathrm{RR}$ \\
\hline _cons & $0.014^{\mathrm{b}}$ & $0.014^{\mathrm{b}}$ & $0.013^{\mathrm{b}}$ & $0.012^{\mathrm{b}}$ & $0.010^{\mathrm{b}}$ \\
\hline $55-59(\operatorname{ref} 50-54)$ & 1.052 & 1.011 & 0.970 & 0.967 & 0.964 \\
\hline $60-64$ & $0.163^{b}$ & $0.160^{\mathrm{b}}$ & $0.163^{\mathrm{b}}$ & $0.164^{\mathrm{b}}$ & $0.164^{b}$ \\
\hline $65-69$ & $0.155^{b}$ & $0.151^{\mathrm{b}}$ & $0.152^{b}$ & $0.153^{\mathrm{b}}$ & $0.152^{b}$ \\
\hline $70-74$ & $0.195^{b}$ & $0.186^{\mathrm{b}}$ & $0.186^{\mathrm{b}}$ & $0.185^{b}$ & $0.184^{b}$ \\
\hline $75-79$ & $0.201^{b}$ & $0.191^{b}$ & $0.191^{b}$ & $0.189^{b}$ & $0.188^{\mathrm{b}}$ \\
\hline $80-84$ & $0.257^{\mathrm{b}}$ & $0.236^{\mathrm{b}}$ & $0.236^{\mathrm{b}}$ & $0.233^{\mathrm{b}}$ & $0.232^{b}$ \\
\hline 85 o más & $0.298^{b}$ & $0.258^{\mathrm{b}}$ & $0.267^{b}$ & $0.264^{b}$ & $0.262^{b}$ \\
\hline Sexo(ref. mujer) & $0.688^{b}$ & $0.670^{\mathrm{b}}$ & $0.640^{\mathrm{b}}$ & $0.617^{\mathrm{b}}$ & $0.660^{\mathrm{b}}$ \\
\hline Nunca unido (ref. unidos) & $1.339^{\mathrm{a}}$ & $1.342^{\mathrm{a}}$ & $1.411^{\mathrm{b}}$ & $1.414^{\mathrm{b}}$ & $1.409^{b}$ \\
\hline Separado, divorciado & $1.168^{\mathrm{a}}$ & $1.172^{b}$ & $1.191^{\mathrm{a}}$ & $1.197^{\mathrm{a}}$ & $1.175^{\mathrm{a}}$ \\
\hline Viudo & $1.488^{b}$ & $1.473^{\mathrm{b}}$ & $1.484^{\mathrm{b}}$ & $1.490^{\mathrm{b}}$ & $1.478^{\mathrm{b}}$ \\
\hline Escolaridad relativa baja (ref. muy baja) & $0.940^{\mathrm{b}}$ & $0.955^{b}$ & $0.940^{\mathrm{b}}$ & $0.941^{b}$ & $0.943^{b}$ \\
\hline Media & $1.066^{\mathrm{b}}$ & $1.105^{\mathrm{b}}$ & $1.107^{\mathrm{b}}$ & $1.110^{\mathrm{b}}$ & $1.110^{\mathrm{b}}$ \\
\hline Alta & $1.292^{\mathrm{a}}$ & $1.317^{\mathrm{b}}$ & $1.293^{\mathrm{b}}$ & $1.298^{\mathrm{b}}$ & $1.302^{\mathrm{b}}$ \\
\hline 1er cuartil (ref. no percibe ingresos) & $0.967^{\mathrm{b}}$ & $0.997^{\mathrm{b}}$ & $0.994^{\mathrm{b}}$ & $0.994^{\mathrm{b}}$ & $0.990^{\mathrm{b}}$ \\
\hline 2do cuartil & $0.967^{\mathrm{b}}$ & $1.000^{\mathrm{b}}$ & $0.977^{\mathrm{b}}$ & $0.983^{b}$ & $0.973^{b}$ \\
\hline 3er cuartil & $0.845^{\mathrm{a}}$ & $0.883^{\mathrm{a}}$ & $0.874^{\mathrm{a}}$ & $0.880^{\mathrm{a}}$ & $0.873^{\mathrm{a}}$ \\
\hline 4to cuartil & $0.630^{\mathrm{b}}$ & $0.654^{\mathrm{b}}$ & $0.643^{\mathrm{b}}$ & $0.651^{\mathrm{b}}$ & $0.644^{\mathrm{b}}$ \\
\hline Regular (ref. excelente, muy buena, buena) & $1.453^{\mathrm{b}}$ & $1.338^{\mathrm{b}}$ & $1.206^{\mathrm{b}}$ & $1.199^{\mathrm{b}}$ & $1.198^{\mathrm{b}}$ \\
\hline Mala & $3.176^{\mathrm{b}}$ & $2.588^{\mathrm{b}}$ & $2.259^{\mathrm{b}}$ & $2.218^{\mathrm{b}}$ & $2.209^{b}$ \\
\hline con dependencia (ref. sin dependencia) & & $1.611^{\mathrm{b}}$ & $1.470^{b}$ & $1.455^{\mathrm{b}}$ & $1.453^{\mathrm{b}}$ \\
\hline $\begin{array}{l}1 \text { enfermedad (ref. ausencia } \\
\text { de enfermedad) }\end{array}$ & & & $1.204^{\mathrm{b}}$ & $1.201^{\mathrm{b}}$ & $1.199^{\mathrm{b}}$ \\
\hline 2 enfermedad & & & $1.657^{\mathrm{b}}$ & $1.647^{\mathrm{b}}$ & $1.635^{\mathrm{b}}$ \\
\hline 3 enfermedad & & & $2.010^{\mathrm{b}}$ & $1.990^{\mathrm{b}}$ & $1.978^{\mathrm{a}}$ \\
\hline 4 enfermedad & & & $1.762^{b}$ & $1.735^{\mathrm{b}}$ & $1.734^{\mathrm{b}}$ \\
\hline Beber (ref. no beber) & & & & $1.160^{\mathrm{b}}$ & $1.181^{\mathrm{b}}$ \\
\hline Fumar (ref. no fumar) & & & & & $1.176^{\mathrm{b}}$ \\
\hline AIC & 37567.1 & 37444.4 & 37320.73 & 37311.88 & 37298.47 \\
\hline BIC & 37694.58 & 37577.91 & 37478.53 & 37475.75 & 37468.42 \\
\hline
\end{tabular}

$\mathrm{N}=3195 .{ }^{\mathrm{a}} \mathrm{p} \leq 0.05 ;{ }^{\mathrm{b}} \mathrm{p} \leq 0.001$

Fuente: elaboración propia a partir de ENASEM. 


\section{RESUlTADOS DEL MODELO DE REGRESIÓN}

El modelo de regresión logístico permite ver los efectos de las variables en conjunto. Al incorporar variables al modelo inicial se puede identificar a través de qué variables se expresa el efecto. Cómo se esperaba, las variables de ingreso y escolaridad no pierden significancia una vez incorporadas las variables de salud. Se muestra así la importancia del nivel socioeconómico para predecir la mortalidad en las edades avanzadas.

El efecto de la edad es significativo, pero se reduce una vez introducidas las otras variables de análisis. Esto no quiere decir que la edad pierda su poder explicativo, sino que existen otros factores que influyen en la supervivencia. Adicionalmente, se observa que los incrementos por grupos de edad no son constantes.

El efecto del sexo es estadísticamente significativo, controlando por las demás variables. Los momios de morir en mujeres es dos tercios la de los hombres, reflejo de la mayor supervivencia femenina. Sin embargo, el tamaño del coeficiente va disminuyendo conforme se van incluyendo otras categorías, indicando que el efecto no es consistente, sino que son otras variables las que van explicando los diferenciales de la supervivencia.

Todas las categorías de situación conyugal son significativas. Las personas nunca unidas son 1.4 veces y las viudas 1.5 veces más propensos a morir que las unidas. La variable escolaridad relativa es una variable estadísticamente significativa cuando es alta. Las personas con escolaridad relativa alta son 1.3 veces más propensos a morir que los de escolaridad relativa baja.

A pesar de incluir todas las variables tanto sociodemográficas como de salud, el ingreso continúa siendo significativo para explicar la supervivencia. Las personas del tercer cuartil tienen momios de 87 por ciento menos de morir respecto a quienes no perciben ingresos y en el cuartil de mayor ingreso los momios son de 64 por ciento menos.

Otros estudios han demostrado la relación fuerte y persistente entre la posición socioeconómica y la mortalidad, pero la investigación reciente sugiere que esta asociación puede ser más débil entre las mujeres. Por otro lado también se oculta la mayor desigualdad en salud, que es más fuerte entre los hombres. Se explica porque los mandatos de la masculinidad impiden sentirse enfermos, asistir al médico y tener un diagnóstico temprano de la enfermedad (Koskinen y Martelein, 1994: 1390).

Esta asociación más débil de la relación mortalidad y nivel socioeconómico por sexo también se ha encontrado en países como Estados Unidos, 
Canadá, Francia, Hungría, Inglaterra y Gales y las naciones nórdicas, donde los diferenciales en salud y en mortalidad son más pronunciados entre hombres que entre mujeres (Liang et al., 2002: 294).

Las categorías del número de enfermedades son estadísticamente significativas. El tener una enfermedad crónica degenerativa no tiene un incremento constante en el riesgo de morir. Quienes presentan una enfermedad son 1.2 veces más propensos a morir que quienes carecen del diagnóstico médico de una enfermedad crónica degenerativa. Cuando son dos enfermedades se incrementa a 1.6 veces y cuando se presentan tres enfermedades la propensión es 1.9 veces mayor que en ausencia de enfermedad. Cabe resaltar que estas cifras podrían estar subestimadas dado que lo que se mide es enfermedad corroborada con diagnóstico médico.

En la variable de auto-percepción de salud todas las categorías son significativas para totales, hombres y mujeres. En el modelo de ambos sexos quienes reportan tener mala salud son 2.2 veces más propensos a morir que quienes reportan tener excelente salud. Quienes tienen al menos una dependencia funcional presentan momios 1.4 veces más de propensión de morir que quienes no presentan dependencia funcional.

Al incorporar la variable de consumo de alcohol los coeficientes de las categorías de escolaridad relativa e ingreso se incrementan en el modelo de totales, hombres y mujeres. Esto podría reflejar un efecto complementario e interrelación entre el nivel socioeconómico y el consumo de alcohol.

A pesar de que la categoría fumar no es significativa para explicar la mortalidad, al incorporar esta variable en el modelo de totales se puede observar que el coeficiente de sexo se reduce, disminuyendo los diferenciales por sexo. Caso contrario ocurre al incorporar la variable beber, pues se amplían los diferenciales por sexo.

En la Figura 1 se puede observar que el efecto multiplicativo de las variaciones en el cuartil de ingreso sobre el riesgo de morir es el mismo independientemente de la edad. Las probabilidades acumuladas de supervivencia alcanzan 0.06 a los 60 años para quienes se sitúan en el cuartil de ingreso más alto, frente a 0.03 para quienes no reportan percibir ingresos, reflejo de las variaciones en el tiempo a la muerte entre personas provenientes de distintos niveles socioeconómicos. La probabilidad en los 80 años se incrementa a 0.06 para quienes no perciben ingreso y quienes están en el cuartil de ingreso más alto la probabilidad es 0.15 . 
Caracteristicas socioeconómicas de supervivientes de 50 o más años de edad en Mexico de 2001 a 2012 / N. ESCALANTE et al.

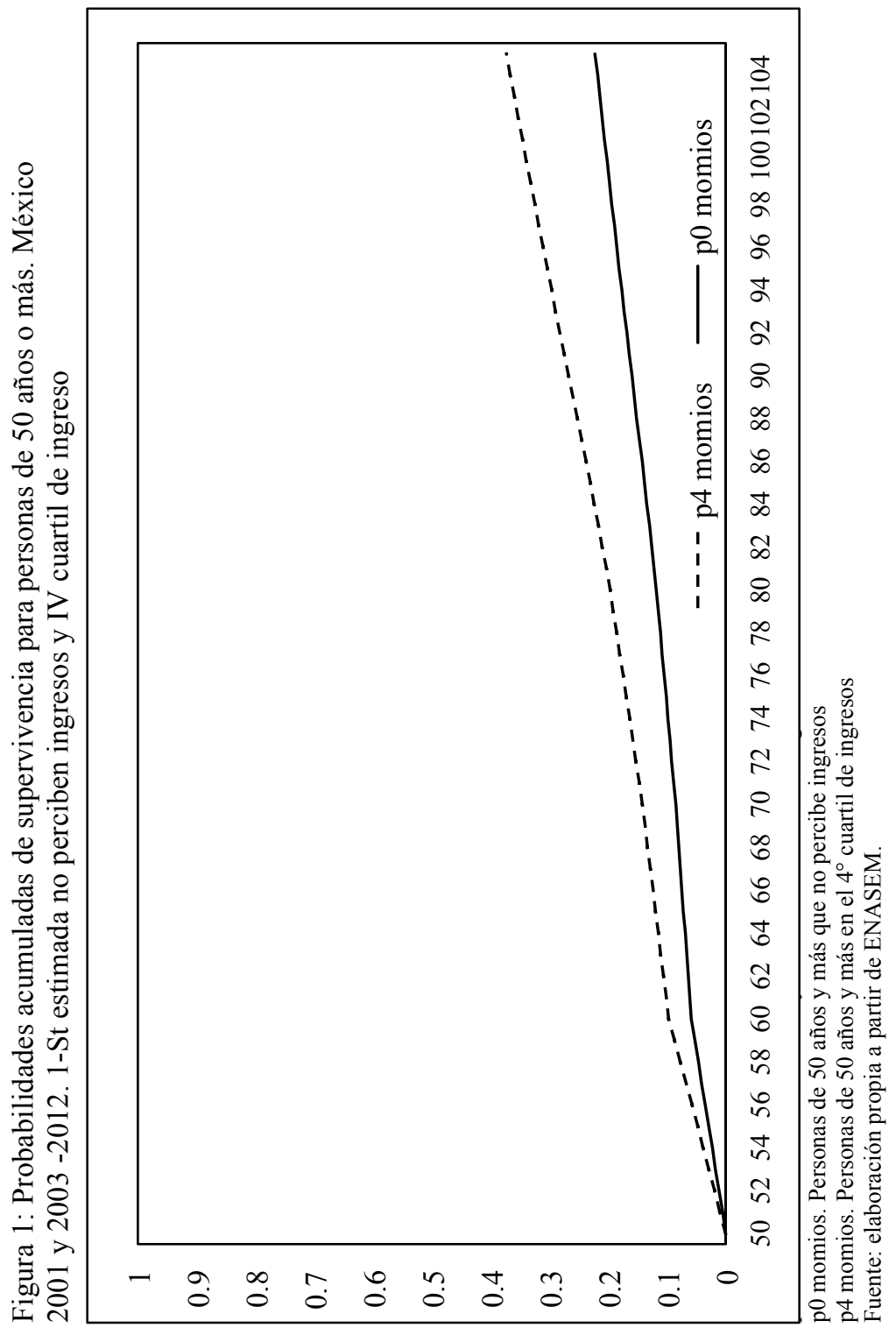


En la Figura 2 se comparan las probabilidades de supervivencia entre grupos de escolaridad relativa, lo que revela un calendario de muerte diferenciado por escolaridad cuando la mayor escolaridad no tiene un efecto protector. La probabilidad acumulada de supervivencia es 0.10 a los 60 años para quienes tienen escolaridad relativa alta, frente a 0.15 para quienes tienen muy baja escolaridad. En la edad 80 años la probabilidad de supervivencia con escolaridad alta es 0.13 frente a 0.20 para quienes tienen escolaridad muy baja. Las diferencias son de mayor magnitud que las observadas en función de la variación entre grupos de ingreso.

El supuesto de proporcionalidad fue verificado en las dos variables que reflejan el nivel socioeconómico: escolaridad relativa e ingreso con respecto a la edad. Los términos de interacción no son significativos en las dos variables relacionadas al nivel socioeconómico de escolaridad relativa e ingreso, por lo que el efecto del ingreso no varía en función del tiempo y esta relación se mantiene después de los 60 años de edad. Como se muestra en los anexos 1a, 1b, 2a y $2 b$.

Si los coeficientes de la interacción fueran positivos el resultado sería el aumento de la desigualdad a medida que incrementa la edad. Si hubieran sido negativos las diferencias entre grupos de ingreso se reducirían y habría convergencia. Pero los resultados muestran que son diferencias relativas constantes en el tiempo y que son equidistantes. ${ }^{2}$

La prueba de hipótesis implica evaluar la significancia estadística de los términos de interacción para todas las combinaciones de grupos de edades y los grupos de ingreso. La interacción no es estadísticamente significativa, lo que hace pensar que el supuesto se está cumpliendo. Esto se puede comprobar mediante una prueba global que contrasta las razones de verosimilitud de ambos modelos: tanto el modelo sin interacciones como el modelo con interacciones.

La prueba formal de razón de verosimilitud confirma el resultado Prob $>\chi^{2}=0.91$. Bajo la hipótesis nula de efectos proporcionales, el estadístico LR $\chi^{2}$ lleva a aceptar $\mathrm{H}_{0}$ y por tanto el supuesto de proporcionalidad de momios. Esto es, que no existe convergencia ni divergencia, sino que las diferencias entre estratos socioeconómicos son estables a medida que se incrementa la edad.

Es deseable que el modelo logístico de tiempo discreto incluya todas las variables independientes relevantes, pero esto casi nunca ocurre debido a la carencia de información.

\footnotetext{
${ }^{2}$ Los anexos 1a, 1b, 2a, 2b muestran la dependencia temporal de los efectos de interacción entre las variables de nivel socioeconómico: escolaridad relativa e ingreso.
} 
Caracteristicas socioeconómicas de supervivientes de 50 o más años de edad en Mexico de 2001 a 2012 / N. ESCALANTE et al.

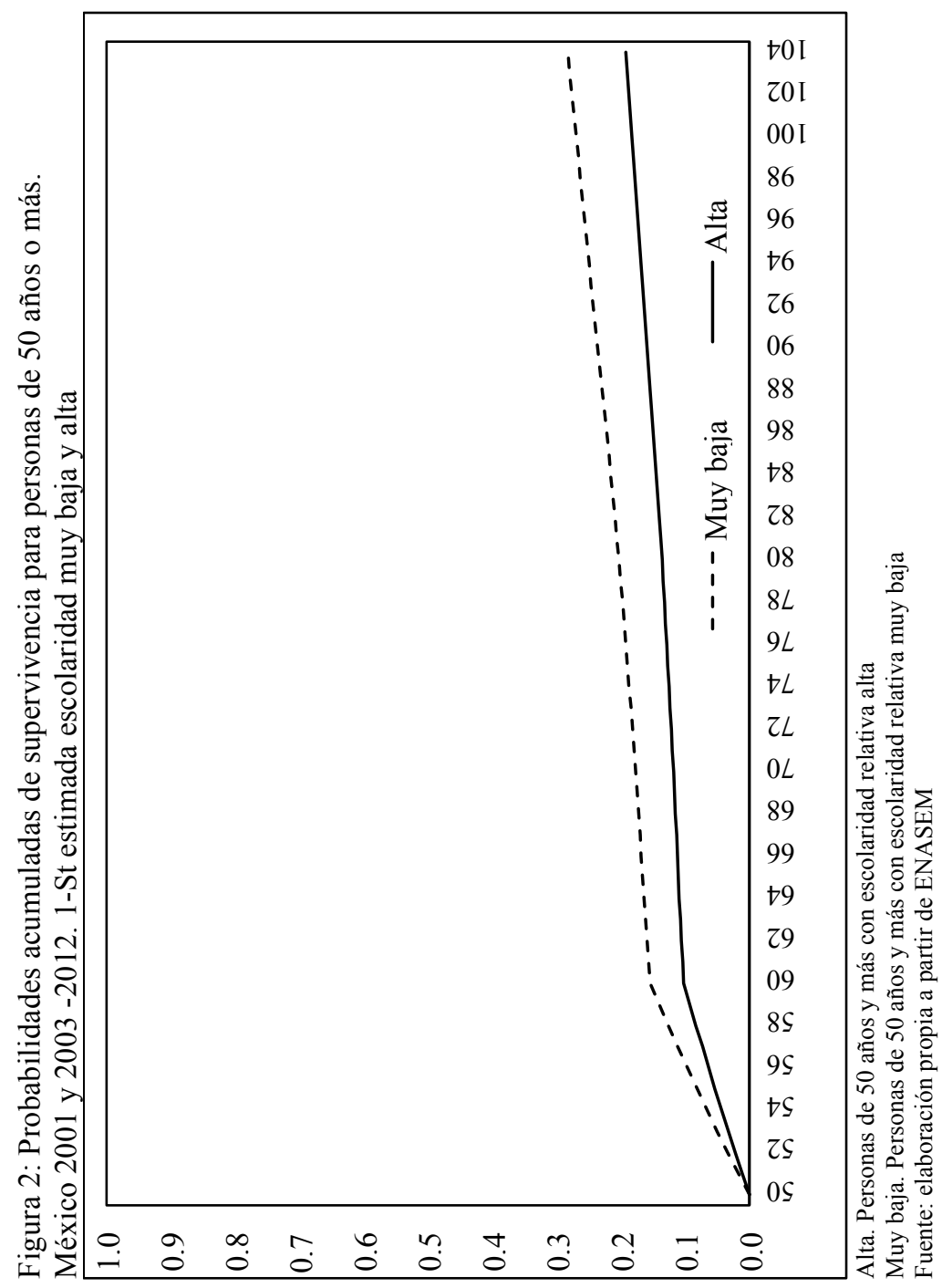


Las consecuencias de la omisión es que en el tiempo los sujetos con ventaja en relación a variables omitidas tenderán a predominar en el conjunto en riesgo, mientras que los sujetos "frágiles" tenderán a ser descartados. La creciente selectividad en el conjunto en riesgo asociada a esta "heterogeneidad no observada" lleva a subestimar el efecto de cualquier otra variable incluida en el modelo, independientemente de si existe o no correlación entre las variables omitidas y la variable dependiente.

Se ha propuesto considerar las variables no observadas. Por ejemplo, la constitución genética, la robustez física, factores ambientales o de estilos de vida o cualesquiera otras variables que hasta ahora no se conocen en relación con la supervivencia. El análisis de la fragilidad representa los efectos combinados de lo desconocido y permite analizar las diferencias en el tiempo de supervivencia entre individuos aparentemente similares. Se introducen términos adicionales en el modelo que da cuenta del grado de "fragilidad" inherente a cada sujeto e independiente de las variables incluidas en el modelo.

Una técnica propuesta para solucionar la heterogeneidad no observada son los modelos de fragilidad (frailty models), que integran términos aleatorios que presuponen la existencia de variables no observables pero que tienen una distribución conocida. La fragilidad se define como multiplicativa de la fuerza de mortalidad y se asume que se mantiene constante a lo largo del incremento de la edad y que no está correlacionada con las variables incluidas en el modelo (Singer y Willet, 2003: 161).

De no ajustarse, la heterogeneidad no observable en los modelos de riesgo pueden sesgar severamente las estimaciones de los efectos estructurales en los modelos de supervivencia (Singer y Willett, 2003: 461). Una vez que se da cuenta de la heterogeneidad no observada entre individuos a partir de este término adicional, se puede obtener estimaciones insesgadas de los efectos de las variables incluidas en el modelo, porque la heterogeneidad no observada podría conducir a la disminución de las diferencias en la mortalidad entre diferentes grupos de edad. Al respecto, Jenkins (1995: 135) propone incorporar un término con distribución gamma cuyos coeficientes se interpretan directamente como una razón de riesgo (hazard rate). ${ }^{3}$

Una hipótesis sobre los efectos del nivel socioeconómico en las probabilidades de edad a la muerte es que al aumentar la edad el efecto del nivel socioeconómico disminuye debido a la selectividad de quienes, proviniendo de sectores con bajos niveles socioeconómicos y en desventaja,

${ }^{3}$ El tamaño de la muestra no permite realizar esta corrección para determinar el efecto de la heterogeneidad. 
sobreviven gracias a factores genéticos o de robustez contra la enfermedad. Esto puede no ser observable, se define como fragilidad, y representa los efectos combinados de otras características que afectan el riesgo a morir.

La Tabla 3 presenta la comparación de las razones de momios de un modelo logístico de tiempo discreto convencional y del modelo con heterogeneidad no observada recién ajustado a partir de una simulación que incorpora un término de fragilidad con una distribución normal. Esto permite conocer en qué medida se alteran las estimaciones y el efecto específico de la heterogeneidad no observable y si ésta es significativa, sobre todo porque la fragilidad oculta procesos de decremento en las desigualdades socioeconómicas y de salud.

El valor de "rho" del modelo ajustado es la razón entre la varianza del término de heterogeneidad no observada y uno más esa misma varianza. Cuando el valor de rho es cero, la heterogeneidad no observada carece de importancia y podemos ajustar un modelo logístico que no incluya el término adicional de fragilidad (Singer y Willett, 2003). En este caso el valor de rho es 0.48 . Se rechaza la hipótesis nula de que $r h o=0$, por lo que concluimos que existe heterogeneidad no observada, es decir, otros factores que influyen en la supervivencia de los adultos en edades avanzadas y selectividad de la mortalidad.

La significancia en este modelo señala la presencia de heterogeneidad no observada, los valores son significativos en casi todas las categorías excepto el grupo de edad 55-59, en las categorías de escolaridad relativa baja y media, en el primer y segundo cuartil de ingresos. En especial interesa el efecto con respecto a las variables de ingreso y escolaridad relativa. $\mathrm{La}$ presencia de heterogeneidad no observada subestima el efecto de la escolaridad y del ingreso con respecto al modelo convencional. Sin embargo, en las categorías de edad no hay efecto sobre la dependencia temporal, sino que se mantiene al incrementarse la edad. En consecuencia, se mantiene la conclusión de las diferencias relativas constantes entre grupos de ingresos y de escolaridad relativa. 
Tabla 3: Comparativo modelo convencional y ajustado con heterogeneidad no observada para personas de 50 años o más. México 2001 y 2003-2012

\begin{tabular}{|c|c|c|c|c|c|}
\hline \multirow{3}{*}{$\begin{array}{l}\text { Variables } \\
\text { Constante }\end{array}$} & \multicolumn{5}{|c|}{ Modelo jerárquico de efectos mixtos } \\
\hline & \multicolumn{2}{|c|}{ Convencional } & \multicolumn{2}{|c|}{ Ajustado } & \multirow{2}{*}{$\begin{array}{r}\text { Diferencia } \\
0.000000\end{array}$} \\
\hline & 0.010 & $* * *$ & 0.010 & $* * *$ & \\
\hline $55-59(\operatorname{ref} 50-54)$ & 0.964 & & 0.964 & & 0.000000 \\
\hline $60-64$ & 0.164 & $* * *$ & 0.164 & *** & 0.000000 \\
\hline $65-69$ & 0.152 & $* * *$ & 0.152 & $* * *$ & 0.000000 \\
\hline $70-74$ & 0.184 & $* * *$ & 0.184 & $* * *$ & 0.000000 \\
\hline $75-79$ & 0.188 & $* * *$ & 0.188 & $* * *$ & 0.000000 \\
\hline $80-84$ & 0.232 & $* * *$ & 0.232 & $* * *$ & 0.000000 \\
\hline 85 o más & 0.262 & $* * *$ & 0.262 & $* * *$ & 0.000000 \\
\hline Sexo(ref. mujer) & 0.660 & *** & 0.660 & $* * *$ & 0.000000 \\
\hline Nunca unido (ref.unidos) & 1.409 & $* * *$ & 1.409 & $* * *$ & -0.000001 \\
\hline Separado, divorciado & 1.175 & ** & 1.175 & ** & 0.000000 \\
\hline Viudo & 1.478 & $* * *$ & 1.478 & $* * *$ & 0.000000 \\
\hline Baja & 0.943 & & 0.943 & & 0.000001 \\
\hline Media & 1.110 & & 1.110 & & -0.000001 \\
\hline Alta & 1.302 & $* * *$ & 1.302 & $* * *$ & -0.000001 \\
\hline 1er cuartil (ref. no percibe ingresos) & 0.990 & & 0.990 & & 0.000001 \\
\hline 2do cuartil & 0.973 & & 0.973 & & 0.000001 \\
\hline 3er cuartil & 0.873 & ** & 0.873 & $* *$ & 0.000000 \\
\hline 4to cuartil & 0.644 & $* * *$ & 0.644 & $* * *$ & 0.000001 \\
\hline $\begin{array}{l}\text { Regular (ref. excelente, muy buena, } \\
\text { buena) }\end{array}$ & 1.198 & $* * *$ & 1.198 & $* * *$ & 0.000000 \\
\hline Mala & 2.209 & $* * *$ & 2.209 & $* * *$ & 0.000003 \\
\hline con dependencia (ref. sin dependencia) & 1.453 & $* * *$ & 1.453 & $* * *$ & 0.000000 \\
\hline $\begin{array}{l}1 \text { enfermedad (ref. ausencia de } \\
\text { enfermedad) }\end{array}$ & 1.199 & $* * *$ & 1.199 & $* * *$ & 0.000000 \\
\hline 2 enfermedad & 1.635 & $* * *$ & 1.635 & $* * *$ & 0.000000 \\
\hline 3 enfermedad & 1.978 & ** & 1.978 & $* * *$ & -0.000001 \\
\hline 4 enfermedad & 1.734 & $* * *$ & 1.734 & ** & 0.000001 \\
\hline Beber (ref. no beber) & 1.181 & $* * *$ & 1.181 & $* * *$ & 0.000000 \\
\hline Fumar (ref. no fumar) & 1.176 & & 1.176 & $* * *$ & -0.000001 \\
\hline
\end{tabular}

** $\mathrm{p} \leq 0.05 ; * * * \mathrm{p} \leq 0.001$.

Fuente: elaboración propia a partir de ENASEM.

\section{ConClusiones}

Es notorio cómo se ha modificado el proceso de envejecimiento manipulando los determinantes sociales y económicos. Es evidente que mucho de lo que explica la supervivencia en edades avanzadas es la exposición a circunstancias socioeconómicas distribuidas de manera desigual entre los 
individuos, lo que repercute en la mortalidad prematura por condiciones adversas.

Este artículo identifica factores que influyen en la supervivencia en edades avanzadas, así como información sobre la dirección y la magnitud de las variables socioeconómicas, demográficas y de salud. Se concluye que la influencia de la escolaridad relativa y el ingreso no varían a medida que se incrementa la edad. En el periodo analizado no hay evidencia de procesos de convergencia e incremento de la desigualdad al avanzar la edad.

Los resultados muestran que los determinantes socioeconómicos explican en gran medida la supervivencia de las personas en edades avanzadas, aun controlando por variables demográficas y de salud. La posición social de ventaja o desventaja relativa del pasado con respecto a otro grupo de ingreso es estable a lo largo de la edad, preservándose la inequidad a lo largo del curso de vida, lo que comprueba parte del enfoque sobre desigualdad acumulada porque la posición relativa se perpetua al largo del tiempo, pero se niega la acumulación.

Las diferencias entre los grupos de ingreso son nulas entre las personas sin ingreso, del primero y del segundo cuartil. Muestra las implicaciones de generar un nivel de vida que esté por lo menos en la media del tercer cuartil de ingreso (alrededor de 3700 pesos mensuales) para tener un efecto en la supervivencia en edades avanzadas. En consecuencia, un objetivo de política social sería aminorar las diferencias socioeconómicas, lo que reduciría necesariamente las diferencias sociales en la mortalidad.

Queda pendiente la discusión de las implicaciones de haber utilizado el ingreso individual y de pareja. Ante la evidencia de que para las personas en edad avanzada en México la corresidencia no implica la socialización de los recursos, habría que discutir la forma en la que se construye la variable hogar, la cual considera la corresidencia, el gasto común en alimentación, la existencia de huéspedes y trabajadores domésticos en el hogar. Sin embargo, otros rubros importantes para este sector de la población, como el gasto en salud y el apoyo ante los procesos de discapacidad y enfermedad, no están considerados. Se requiere un mayor análisis para determinar las implicaciones que tiene utilizar el ingreso individual versus el ingreso relativo del hogar en la construcción del nivel socioeconómico y en qué medida afectan la supervivencia.

La hipótesis de nivelación queda descartada porque ningún coeficiente de la interacción entre las variables de ingreso y la edad es negativo o decreciente. La edad como nivelador social habría requerido suponer, ya sea que los individuos con mayor ingreso experimentan un descenso más rápi- 
do en la salud y muerte a través de la edad que aquellos con bajos niveles de ingreso, o bien que la salud mejora más rápidamente con el incremento de la edad para las personas con bajos niveles de ingreso que para los que tienen altos niveles de ingreso. Ambas son expectativas poco razonables.

Otro razonamiento a la luz de haber encontrado diferencias relativas constantes entre diferentes grupos de ingreso, lleva a pensar que la disminución de la condición de salud por el proceso de envejecimiento al avanzar la edad se distribuye en partes iguales entre los diferentes niveles socioeconómicos, lo suficiente como para dar lugar a estabilidad.

Los resultados se circunscriben a la población de 50 años o más. Sin embargo, cabe preguntarse si la comparación entre edades anteriores a ésta última provocaría un cambio en las conclusiones, sobre todo al considerar que la mayoría de los jóvenes están sanos y por lo tanto presentan menos variabilidad en la salud en edades más tempranas. Al incorporar en el análisis todos los grupos de edad habría que considerar que el efecto de la edad tiene una forma funcional cuadrática y que podría estar generando procesos de convergencia en los extremos de las edades y un incremento en la edad media.

La relación persistente entre la posición socioeconómica y la supervivencia en edades avanzadas sugiere que es más débil para las mujeres, lo cual se explica porque son más semejantes a la baja en su condición de ingreso, pero también oculta la mayor desigualdad en salud para los hombres, lo que explica la mayor desigualdad entre ellos en los coeficientes de salud.

Los resultados presentados difieren de los estudios mencionados en los antecedentes porque todos pertenecen a países con distintas situaciones sociales, políticas, económicas y culturales y, sobre todo, porque ninguno se ha hecho en América Latina. También se da el factor metodológico que toma en consideración el incremento de la escolaridad que es muy importante en México. Para evitar que el efecto del incremento de la escolaridad provoque procesos de convergencia en edades avanzadas por la reducción en la brecha por escolaridad de los distintos grupos sociales, se controló a partir de la construcción de la variable de "escolaridad relativa" que controla por edad, sexo y cohorte de nacimiento.

La presente investigación considera el efecto en el incremento del número de años de la escolaridad con respecto a la cohorte y sexo de nacimiento, pero también sería importante considerar el efecto de la calidad de los contenidos educativos en la supervivencia en edades avanzadas. 
Las principales diferencias por situación conyugal muestran que las personas unidas tienen menor propensión a morir que en las otras categorías. Esta relación también se corrobora en Estados Unidos y Japón. Al parecer el matrimonio tiene un efecto protector, aunque se requiere mayor investigación para determinar si es un efecto asociado directamente al matrimonio o si es un mecanismo de selección (Liang et al., 2002: 301). También se debe determinar el impacto en la población mexicana por el número de años expuestos a las distintas situaciones conyugales. Por su parte, los hombres divorciados y separados tienen un mayor riesgo a morir en contraste con las mujeres en la misma situación.

Los hábitos de salud muestran un fuerte impacto en las posibilidades de fallecer y reducen las diferencias por sexo, pero incrementan las diferencias por ingreso. El riesgo de muerte por bebidas alcohólicas es mayor entre mujeres que entre hombres, dando importancia a los hábitos de salud en la supervivencia por sexo.

La discusión del nivel socioeconómico y su relación con el incremento de edad fue analizada a partir de un modelo que incorpora la presencia de heterogeneidad no observable, porque algunos individuos tienen un mayor riesgo de morir relativamente antes, lo que provoca selectividad y sesgos en la relación de la variación del efecto del predictor en el tiempo. El modelo muestra que la relación de estabilidad del nivel socioeconómico en el tiempo se mantiene.

Finalmente, es necesario señalar la importancia de seguir generando información de tipo longitudinal que permita profundizar en los efectos de los cambios en las variables sociodemográficas sobre la supervivencia en las edades avanzadas. Cualquier avance en la comprensión de la importancia de la situación socioeconómica de la salud requerirá datos longitudinales, aunque implique décadas de seguimiento.

Generar este tipo de información permitirá contrastar los cambios entre cohortes a la luz de procesos demográficos como la reducción en las tasas de fecundidad y el aumento en las tasas de divorcio. Se deben también considerar otros cambios como la apertura comercial, el incremento de la informalidad, el incremento del empleo femenino que disminuye la posibilidad de tiempo por parte de las mujeres para cuidar personas en la vejez, la mayor rotación laboral y todo lo que dé lugar a inestabilidad y pobreza. Asimismo, se debe evaluar el efecto de mayor escolaridad y calidad del sistema educativo, como una transformación que favorece la supervivencia. 
Anexo

Anexo 1: Modelo Interacción edad y cuartiles de ingreso

\begin{tabular}{|c|c|c|c|c|c|c|c|}
\hline \multicolumn{2}{|l|}{ Edad } & \multirow{2}{*}{$\begin{array}{r}\begin{array}{r}\text { Razón de } \\
\text { momios }\end{array} \\
0.968\end{array}$} & \multirow{2}{*}{$\begin{array}{r}\text { Std. } \\
\text { Err. } \\
0.303\end{array}$} & \multirow[b]{2}{*}{-0 100} & \multirow{2}{*}{$\begin{array}{c}P>z \\
0.917\end{array}$} & \multicolumn{2}{|c|}{$\begin{array}{c}95 \% \\
\text { Intervalo Conf. }\end{array}$} \\
\hline \multicolumn{2}{|c|}{$55-59($ ref $50-54)$} & & & & & 0.524 & 1.788 \\
\hline \multicolumn{2}{|c|}{$60-64$} & 0.227 & 0.066 & -5.070 & 0.000 & 0.128 & 0.402 \\
\hline \multicolumn{2}{|l|}{$65-69$} & 0.185 & 0.053 & -5.840 & 0.000 & 0.105 & 0.326 \\
\hline \multicolumn{2}{|l|}{$70-74$} & 0.271 & 0.076 & -4.650 & 0.000 & 0.156 & 0.469 \\
\hline \multicolumn{2}{|l|}{$75-79$} & 0.251 & 0.071 & -4.880 & 0.000 & 0.144 & 0.437 \\
\hline \multicolumn{2}{|l|}{$80-84$} & 0.302 & 0.085 & -4.270 & 0.000 & 0.174 & 0.523 \\
\hline \multicolumn{2}{|l|}{85 y más } & 0.344 & 0.096 & -3.840 & 0.000 & 0.200 & 0.593 \\
\hline $55-59$ & $1^{\mathrm{er}}$ cuartil & 0.918 & 0.474 & -0.170 & 0.868 & 0.334 & 2.524 \\
\hline $55-59$ & $2^{\text {do }}$ cuartil & 1.058 & 0.508 & 0.120 & 0.907 & 0.413 & 2.714 \\
\hline $55-59$ & $3^{\text {er }}$ cuartil & 0.933 & 0.420 & -0.150 & 0.878 & 0.386 & 2.257 \\
\hline $55-59$ & $4^{\text {to }}$ cuartil & 1.087 & 0.470 & 0.190 & 0.848 & 0.465 & 2.537 \\
\hline $60-64$ & $1^{\mathrm{er}}$ cuartil & 0.705 & 0.327 & -0.750 & 0.450 & 0.284 & 1.749 \\
\hline $60-64$ & $2^{\text {do }}$ cuartil & 1.047 & 0.472 & 0.100 & 0.919 & 0.432 & 2.534 \\
\hline $60-64$ & $3^{\text {er }}$ cuartil & 0.566 & 0.243 & -1.330 & 0.185 & 0.243 & 1.314 \\
\hline $60-64$ & $4^{\text {to }}$ cuartil & 0.433 & 0.178 & -2.040 & 0.062 & 0.193 & 0.969 \\
\hline $65-69$ & $1^{\mathrm{er}}$ cuartil & 0.752 & 0.345 & -0.620 & 0.534 & 0.306 & 1.847 \\
\hline $65-69$ & $2^{\text {do }}$ cuartil & 1.114 & 0.497 & 0.240 & 0.808 & 0.465 & 2.671 \\
\hline $65-69$ & $3^{\text {er }}$ cuartil & 0.718 & 0.307 & -0.780 & 0.438 & 0.311 & 1.658 \\
\hline $65-69$ & $4^{\text {to }}$ cuartil & 0.580 & 0.236 & -1.340 & 0.180 & 0.261 & 1.287 \\
\hline $70-74$ & $1^{\mathrm{er}}$ cuartil & 0.599 & 0.270 & -1.140 & 0.256 & 0.248 & 1.449 \\
\hline $70-74$ & $2^{\text {do }}$ cuartil & 0.826 & 0.363 & -0.440 & 0.663 & 0.349 & 1.955 \\
\hline $70-74$ & $3^{\text {er }}$ cuartil & 0.563 & 0.238 & -1.360 & 0.174 & 0.246 & 1.289 \\
\hline $70-74$ & $4^{\text {to }}$ cuartil & 0.392 & 0.162 & -2.270 & 0.063 & 0.175 & 0.879 \\
\hline $75-79$ & $1^{\mathrm{er}}$ cuartil & 0.614 & 0.277 & -1.080 & 0.280 & 0.253 & 1.487 \\
\hline $75-79$ & $2^{\text {do }}$ cuartil & 0.933 & 0.411 & -0.160 & 0.875 & 0.393 & 2.214 \\
\hline $75-79$ & $3^{\text {er }}$ cuartil & 0.678 & 0.289 & -0.910 & 0.363 & 0.294 & 1.565 \\
\hline $75-79$ & 4to cuartil & 0.592 & 0.243 & -1.280 & 0.202 & 0.265 & 1.324 \\
\hline $80-84$ & $1^{\mathrm{er}}$ cuartil & 0.681 & 0.306 & -0.860 & 0.392 & 0.282 & 1.644 \\
\hline $80-84$ & $2^{\text {do }}$ cuartil & 0.931 & 0.410 & -0.160 & 0.870 & 0.393 & 2.205 \\
\hline $80-84$ & $3^{\text {er }}$ cuartil & 0.677 & 0.293 & -0.900 & 0.367 & 0.290 & 1.580 \\
\hline $80-84$ & $4^{\text {to }}$ cuartil & 0.646 & 0.274 & -1.030 & 0.303 & 0.282 & 1.483 \\
\hline 85 o más & $1^{\mathrm{er}}$ cuartil & 0.629 & 0.280 & -1.040 & 0.298 & 0.263 & 1.505 \\
\hline 85 o más & $2^{\text {do }}$ cuartil & 0.942 & 0.412 & -0.140 & 0.891 & 0.400 & 2.218 \\
\hline 85 o más & $3^{\text {er }}$ cuartil & 0.736 & 0.314 & -0.720 & 0.473 & 0.319 & 1.700 \\
\hline 85 o más & $4^{\text {to }}$ cuartil & 0.656 & 0.274 & -1.010 & 0.312 & 0.290 & 1.486 \\
\hline $\begin{array}{l}1^{\text {er }} \text { cuartil } \\
\text { percibe in }\end{array}$ & $\begin{array}{l}\text { ef. no } \\
\text { esos) }\end{array}$ & 1.486 & 0.642 & 0.920 & 0.359 & 0.637 & 3.466 \\
\hline $2^{\text {do }}$ cuartil & & 1.018 & 0.428 & 0.040 & 0.965 & 0.447 & 2.320 \\
\hline $3^{\text {er }}$ cuartil & & 1.308 & 0.520 & 0.670 & 0.500 & 0.599 & 2.853 \\
\hline $4^{\text {to }}$ cuartil & & 1.132 & 0.420 & 0.330 & 0.738 & 0.547 & 2.341 \\
\hline Sexo (ref. & nujer) & 0.661 & 0.030 & -9.150 & 0.000 & 0.605 & 0.723 \\
\hline $\begin{array}{l}\text { Nunca un } \\
\text { unidos) }\end{array}$ & (ref. & 1.412 & 0.134 & 3.620 & 0.000 & 1.171 & 1.701 \\
\hline Separado, & vorciado & 1.181 & 0.085 & 2.300 & 0.022 & 1.025 & 1.361 \\
\hline Viudo & & 1.475 & 0.071 & 8.100 & 0.000 & 1.343 & 1.620 \\
\hline $\begin{array}{l}\text { Escolarid: } \\
\text { baja (ref. }\end{array}$ & $\begin{array}{l}\text { relativa } \\
\text { Muy baja) }\end{array}$ & 0.951 & 0.056 & -0.850 & 0.393 & 0.847 & 1.067 \\
\hline Media & & 1.114 & 0.075 & 1.610 & 0.107 & 0.977 & 1.270 \\
\hline Alta & & 1.306 & 0.090 & 3.870 & 0.000 & 1.141 & 1.496 \\
\hline Regular ( & & & & & & & \\
\hline $\begin{array}{l}\text { Excelente } \\
\text { Buena) }\end{array}$ & Muy buena, & 1.192 & 0.058 & 3.640 & 0.000 & 1.085 & 1.311 \\
\hline Mala & & 2.204 & 0.182 & 9.570 & 0.000 & 1.875 & 2.592 \\
\hline $\begin{array}{l}\text { Con deper } \\
\text { Sin depen }\end{array}$ & $\begin{array}{l}\text { encia (ref. } \\
\text { encia) }\end{array}$ & 1.453 & 0.062 & 8.690 & 0.000 & 1.335 & 1.581 \\
\hline 1 enferme & d (ref. & & & & & & \\
\hline $\begin{array}{l}\text { Ausencia } \\
\text { enfermeda }\end{array}$ & & 1.195 & 0.056 & 3.790 & 0.000 & 1.090 & 1.310 \\
\hline 2 enferme & ades & 1.629 & 0.087 & 9.190 & 0.000 & 1.468 & 1.808 \\
\hline 3 enferm & ades & 1.967 & 0.152 & 8.730 & 0.000 & 1.690 & 2.290 \\
\hline 4 enferme & des & 1.718 & 0.302 & 3.080 & 0.002 & 1.217 & 2.424 \\
\hline Beber (re & No beber) & 1.174 & 0.049 & 3.860 & 0.000 & 1.082 & 1.273 \\
\hline Fumar (re & No fumar) & 1.177 & 0.054 & 3.550 & 0.000 & 1.076 & 1.288 \\
\hline Constante & & 0.008 & 0.002 & 17.530 & 0.000 & 0.005 & 0.014 \\
\hline
\end{tabular}


Características socioeconómicas de supervivientes de 50 omás años de edad en México de 2001 a 2012 / N. ESCALANTE et al.

Anexo 2: Modelo Interacción edad y escolaridad relativa

\begin{tabular}{|c|c|c|c|c|c|c|c|}
\hline \multicolumn{2}{|l|}{ Edad } & $\begin{array}{l}\text { Odds } \\
\text { Ratio }\end{array}$ & $\begin{array}{l}\text { Std. } \\
\text { Err. }\end{array}$ & $\mathrm{z}$ & $\mathrm{P}>\mathrm{Z}$ & $95 \%$ Conf. & terval \\
\hline \multicolumn{2}{|c|}{ 55-59 (ref. 50-54) } & 0.907 & 0.158 & -0.560 & 0.574 & 0.644 & 1.277 \\
\hline \multicolumn{2}{|c|}{$60-64$} & 0.152 & 0.025 & -11.440 & 0.000 & 0.110 & 0.210 \\
\hline \multicolumn{2}{|l|}{$65-69$} & 0.138 & 0.022 & -12.150 & 0.000 & 0.100 & 0.190 \\
\hline \multicolumn{2}{|l|}{$70-74$} & 0.182 & 0.029 & -10.570 & 0.000 & 0.132 & 0.249 \\
\hline \multicolumn{2}{|l|}{$75-79$} & 0.185 & 0.030 & -10.400 & 0.000 & 0.135 & 0.255 \\
\hline \multicolumn{2}{|l|}{$80-84$} & 0.217 & 0.035 & -9.400 & 0.000 & 0.158 & 0.298 \\
\hline \multicolumn{2}{|l|}{85 o más } & 0.248 & 0.040 & -8.660 & 0.000 & 0.181 & 0.340 \\
\hline \multicolumn{2}{|c|}{$\begin{array}{l}\text { Escolaridad relativa baja (ref. } \\
\text { Muy baja) }\end{array}$} & 0.813 & 0.301 & -0.560 & 0.577 & 0.393 & 1.682 \\
\hline \multicolumn{2}{|c|}{ Media } & 1.104 & 0.665 & 0.160 & 0.870 & 0.339 & 3.593 \\
\hline \multicolumn{2}{|l|}{ Alta } & 0.893 & 0.472 & -0.210 & 0.831 & 0.317 & 2.518 \\
\hline $55-59$ & Baja & 1.193 & 0.507 & 0.410 & 0.678 & 0.519 & 2.742 \\
\hline $60-64$ & Baja & 1.471 & 0.582 & 0.980 & 0.329 & 0.678 & 3.193 \\
\hline $65-69$ & Baja & 1.421 & 0.562 & 0.890 & 0.374 & 0.654 & 3.087 \\
\hline $70-74$ & Baja & 0.882 & 0.348 & -0.320 & 0.751 & 0.407 & 1.911 \\
\hline $75-79$ & Baja & 0.735 & 0.304 & -0.740 & 0.456 & 0.327 & 1.653 \\
\hline $80-84$ & Baja & 1.312 & 0.549 & 0.650 & 0.516 & 0.578 & 2.981 \\
\hline 85 y más & Baja & 1.445 & 0.576 & 0.920 & 0.356 & 0.662 & 3.158 \\
\hline $55-59$ & Media & 1.087 & 0.720 & 0.130 & 0.900 & 0.297 & 3.980 \\
\hline $60-64$ & Media & 1.096 & 0.704 & 0.140 & 0.886 & 0.311 & 3.861 \\
\hline $65-69$ & Media & 1.195 & 0.753 & 0.280 & 0.777 & 0.348 & 4.106 \\
\hline $70-74$ & Media & 0.945 & 0.590 & -0.090 & 0.927 & 0.278 & 3.214 \\
\hline $75-79$ & Media & 1.075 & 0.666 & 0.120 & 0.907 & 0.319 & 3.623 \\
\hline $80-84$ & Media & 0.976 & 0.606 & -0.040 & 0.969 & 0.289 & 3.295 \\
\hline 85 o más & Media & 0.855 & 0.532 & -0.250 & 0.801 & 0.252 & 2.895 \\
\hline $55-59$ & Alta & 1.408 & 0.853 & 0.560 & 0.572 & 0.429 & 4.619 \\
\hline $60-64$ & Alta & 1.021 & 0.593 & 0.040 & 0.972 & 0.327 & 3.190 \\
\hline $65-69$ & Alta & 1.556 & 0.879 & 0.780 & 0.434 & 0.514 & 4.706 \\
\hline $70-74$ & Alta & 1.734 & 0.962 & 0.990 & 0.321 & 0.584 & 5.145 \\
\hline $75-79$ & Alta & 1.417 & 0.784 & 0.630 & 0.529 & 0.479 & 4.192 \\
\hline $80-84$ & Alta & 1.621 & 0.895 & 0.870 & 0.382 & 0.549 & 4.784 \\
\hline 85 y más & Alta & 1.442 & 0.784 & 0.670 & 0.501 & 0.497 & 4.184 \\
\hline $\begin{array}{l}1^{\mathrm{er}} \text { cuartil } \\
\text { percibe in }\end{array}$ & & 0.994 & 0.052 & -0.120 & 0.904 & 0.896 & 1.101 \\
\hline $2^{\text {do }}$ cuartil & & 0.976 & 0.053 & -0.450 & 0.652 & 0.876 & 1.086 \\
\hline $3^{\text {er }}$ cuartil & & 0.878 & 0.053 & -2.150 & 0.031 & 0.779 & 0.988 \\
\hline $4^{\text {to }}$ cuartil & & 0.641 & 0.046 & -6.150 & 0.000 & 0.556 & 0.739 \\
\hline Sexo(ref. & & 0.651 & 0.029 & -9.520 & 0.000 & 0.596 & 0.711 \\
\hline Nunca un & ef. Unidos) & 1.409 & 0.134 & 3.600 & 0.000 & 1.169 & 1.698 \\
\hline Separado, & rciado & 1.175 & 0.085 & 2.230 & 0.026 & 1.020 & 1.354 \\
\hline Viudo & & 1.479 & 0.071 & 8.180 & 0.000 & 1.347 & 1.625 \\
\hline $\begin{array}{l}\text { Regular }(1 \\
\text { buena, } \mathrm{Bu}\end{array}$ & xcelente, Muy & 1.205 & 0.058 & 3.840 & 0.000 & 1.096 & 1.325 \\
\hline Mala & & 2.216 & 0.183 & 9.640 & 0.000 & 1.885 & 2.605 \\
\hline $\begin{array}{l}\text { Con deper } \\
\text { dependen }\end{array}$ & ia (ref. Sin & 1.458 & 0.063 & 8.780 & 0.000 & 1.340 & 1.586 \\
\hline $\begin{array}{l}1 \text { enferme } \\
\text { de enferm }\end{array}$ & ref. Ausencia & 1.197 & 0.056 & 3.830 & 0.000 & 1.092 & 1.313 \\
\hline 2 enferme & & 1.628 & 0.086 & 9.200 & 0.000 & 1.468 & 1.806 \\
\hline 3 enferme & & 1.968 & 0.152 & 8.740 & 0.000 & 1.691 & 2.291 \\
\hline 4 enferme & & 1.717 & 0.301 & 3.080 & 0.002 & 1.217 & 2.421 \\
\hline Beber (re & beber) & 1.179 & 0.049 & 3.980 & 0.000 & 1.087 & 1.279 \\
\hline Fumar (r & o fumar) & 1.182 & 0.054 & 3.640 & 0.000 & 1.080 & 1.293 \\
\hline Constante & & 0.011 & 0.002 & -27.190 & 0.000 & 0.008 & 0.015 \\
\hline
\end{tabular}




\section{REFERENCIAS BIBLIOGRÁFICAS}

Echarri, Carlos, 2008, "Desigualdad socioeconómica y salud reproductiva: una propuesta de estratificación social aplicable a las encuestas", en Lerner, Susana y Szasz, Ivonne (coords.), Salud reproductiva y condiciones de vida en México, Tomo I, Colegio de México, pp. 59-113. México.

Hoffman, Rasmus, 2008, "Socioeconomic differences in old age mortality", in The Springer Series on Demographic Methods and Population Analysis, vol. 25, pp. 244. Alemania.

House, James; Lepkowski, James; Kinney, Ann, Mero, Richard; Kessler, Ronald y Herzog, Regula, 1994, "The social stratification of aging and health", en Journal of health and social behavior, vol. 35, núm. 3, 7 de junio 2015, pp. 213-234. Disponible en http://www.jstor.org/stable/2137277

Jenkins, Stephen, 1995, "Easy estimation methods for discrete-time duration models", in Oxford Bulletin of Economics and Statistics, vol. 57, núm. 1, 7 de junio 2015, pp. 129-136 DOI: 10.1111/j.1468-0084.1995.tb00031.x

Koskinen, Sepfq y Martelein, Tuija, 1994, Why are Studies socioeconomic mortality differences smaller among women than among men?, in Social Science and Medicine, vol. 38, núm. 10, 7 de junio 2015, pp. 1385-1396. Disponible en https:// www.ncbi.nlm.nih.gov/pubmed/8023188

Lauderdale, Diane, 2001, "Education and survival: birth cohort, period, and age effects", in Demography, vol. 38, núm. 4, 7 de junio 2015, pp. 551-561. Disponible en http://www.jstor.org/stable/3088318

Liang, Jersey; Bennett, Joan; Krause, Neal; Kobayashi, Erika; Hyekyung, Kim; Winchester, Brown; Hiroko, Akiyama; Hidehiro, Sugisawa y Jain, Arvind, 2002, "Old Age Mortality in Japan: Does the Socioeconomic Gradient Interact With Gender and Age?", in The Journals of Gerontology Series B: Psychological Sciences and Social Sciences, vol. 57, núm. 5, 7 de junio 2015, pp. 294-307. DOI: 10.1093/geronb/57.5.S294

Lynch, Scott, 2003, "Cohort and life-course patterns in the relationship between education and health: a hierarchical approach", in Demography, vol. 40, núm. 2, 7 de junio 2015, pp. 309-331. Disponible en http://www.jstor.org/stable/3180803

Marmot, Michael y Shipley, Martin, 1996, "Do socioeconomic differences in mortality persist after retirement? 25 year follow up of civil servants from the first Whitehall study", in British Medical Journal, vol. 313, núm. 7066, 7 de junio 2015, pp. 1177-1180. Disponible en: http://www.popline.org/node/302393

Mayer, Karl y Wagner, Michael, 1993, "Socio-economic resources and differential ageing", in Ageing and Society, vol. 13, núm. 4, 7 de junio 2015, pp. 517-550. DOI: $10.1017 / \mathrm{S} 0144686 \mathrm{X} 00001355$

O'Rand, Angela, 1996, "The precious and the precocious: understanding cumulative disadvantage and cumulative advantage over the life course", in The Geron- 
tologist, vol. 36, núm. 2, 7 de junio 2015, pp. 230-238. DOI: por ciento10.1093/ geront/36.2.230

Pampel, Fred y Hardy, Melissa, 1994, "Status maintenance and change during old age”, in Social Forces, vol. 73, núm. 1, 7 de junio 2015, pp. 289-314. Disponible en http://www.jstor.org/stable/2579927

Prus, Steven, 2004, "A life course perspective on the relationship between socio-economic status and health: por cientotesting the divergence hypothesis", in Canadian Journal on Aging, vol. 23, núm. 1, 7 de junio 2015, pp. S145-S153. Disponible en https://muse.jhu.edu/article/182903/pdf

Ross, Catherine y Wu, Chia-Ling, 1996, "Education, Age, and the Cumulative Advantage in Health", in Journal of Health and Social Behavior, vol. 37, núm. 1, 7 de junio 2015, pp. 104-120. Disponible en http://www.jstor.org/stable/2137234

Singer, Judith y Willett, John, 2003, Applied longitudinal data analysis, Oxford University Press, Oxford.

Vaupel, James, 2001, "Mortality of the Oldest Old", in International Encyclopedia of Social and Behavioral Sciences, 10075-10079.

\section{DAtos CURRICUlares de LOS AUTORES}

Nancy Escalante Rivas

Licenciada en Sociología por la Facultad de Ciencias Políticas y Sociales, UNAM y Maestra en Demografía por El Colegio de México. Profesora del Sistema Universidad Abierta y Educación a Distancia de la Facultad de Ciencias Políticas y Sociales, UNAM.

Dirección electrónica: nancy-escalante@hotmail.com

\section{Roberto Ham-Chande}

Actuario de la Facultad de Ciencias, UNAM. Maestría en Estadística Matemática, Centro Interamericano de Enseñanza de Estadística, Santiago, Chile. Maestría en Ciencias, Department of Statistics and Probability, Michigan State University. Doctor en Demografía, Université de Paris Nanterre, con la máxima mención honorífica. Es profesor-investigador del Departamento de Estudios en Población en El Colegio de la Frontera Norte y profesor honorario de El Colegio de México. Ha sido actuario del IMSS y del ISSSTE. Ex-director del Centro de Estudios Demográficos y Desarrollo Urbano de El Colegio de México. Pertenece al Sistema Nacional de Investigadores desde 1985, actualmente en nivel 3. Premio Nacional de Demografía 2009.

Dirección electrónica: rham@colef.mx 
Carlos Javier Echarrí Canovas

Profesor Investigador. Centro de Estudios Demográficos, Urbanos y Ambientales (CEDUA), El Colegio de México. Actuario por la Facultad de Ciencias de la UNAM. Maestro en Demografía por El Colegio de México. Doctor en Demografía por la Universidad católica de Lovaina, Bélgica. Investigador nacional, nivel II. Coordinador del Programa Salud Reproductiva y Sociedad, El Colegio de México. Coordinador del Cuerpo Académico Reproducción de la Población y Salud, PROMEP. Miembro del Comité Científico Asesor del Diagnóstico Nacional sobre violencia contra las mujeres CRIM/UNAM/CONAVIM. Vocal del Comité de Prevención, del Consejo Nacional para la Prevención y Control del SIDA CONASIDA. Dirección electrónica: cecha@colmex.mx

Artículo recibido el 5 de noviembre de 2016 y aprobado el 10 de abril de 2017. 